\title{
10 Magnetic characterization of magnetoactive elastomers containing magnetic hard particles using first-order reversal curve analysis
}

\begin{abstract}
The use of new types of intelligent materials is becoming increasingly widespread. These include magnetoactive elastomers with hard magnetic filling components, which offer the unique chance to adapt active and passive material properties. In this context, this paper presents an overview of the experimental results on the study of the magnetic properties of elastic composites with a magnetic hard component. First-order reversal curves, which are recorded with a vibrating sample magnetometer, are used as method to characterize the magnetic material behavior. The influence of various parameters on the process of magnetization of composites is considered, including the stiffness of the polydimethylsiloxane-based matrix polymer, the particle ratio and the particle size as well as the so-called training effect.
\end{abstract}

Keywords: first-order reversal curve, interparticle interaction, magnetization behavior, smart materials

\subsection{Introduction}

Magnetic hybrid materials are a kind of smart materials consisting of a matrix ranging from Newtonian liquids to polymerized elastomers and a magnetic filler, i.e., magnetic particles which can be micron-as well as nanosized, and which can be made of magnetically soft or hard materials [1]. A prominent example of a material within this class of magnetic hybrid composites are magnetic particles embedded in a polymeric matrix. Important for the design of applications using magnetic hybrid materials is a strong change in the respective properties induced by a magnetic field. This necessity requires a high-particle volume fraction and a low elastic modulus of the matrix. Magnetic hybrid materials basied on such soft matrices are usually called soft magnetic elastomers as well as magnetoactive elastomers (MAEs). Caused by external stimuli, such as external magnetic fields, the physical properties of MAEs can be controlled and adapted to the specific application [2].

\footnotetext{
*Corresponding authors: Stefan Odenbach and Mia Schliephake, TU Dresden, Faculty of Mechanical Engineering, George-Bähr-Str. 3, 01062 Dresden, Germany, E-mail: stefan.odenbach@tu-dresden.de (S. Odenbach), mia.schliephake@tu-dresden.de (M. Schliephake)

Julia M. Linke, TU Dresden, Faculty of Mechanical Engineering, George-Bähr-Str. 3, 01062 Dresden, Germany

O Open Access. (c) 2021 Mia Schliephake et al., published by De Gruyter. (c) BY-NC-ND This work is licensed under the Creative Commons Attribution-NonCommercial-NoDerivatives 4.0 International License.

This article has previously been published in the journal Physical Sciences Reviews. Please cite as: M. Schliephake, J. M. Linke and S. Odenbach "Magnetic characterization of magnetoactive elastomers containing magnetic hard particles using first-order reversal curve analysis" Physical Sciences Reviews [Online] 2021, 6. DOI: 10.1515/psr-2019-0124 | https://doi.org/10.1515/ 9783110569636-010
} 
Conventionally, MAEs use magnetically soft particles providing a magnetic control due to external fields. This is usually called a magnetoactive material [3]. Alternatively, magnetically hard particles can be used as a filler, which lead to passive magnetorheological properties of the composite [4]. It has also been shown that mixtures of magnetically hard and soft particles lead to new properties of the corresponding MAEs, which are not only a simple superposition of the passive and active effects but also which would be found in elastomers containing hard or soft particles only [5-7]. The overall properties of such mixed MAEs rely obviously on the complex interaction of the magnetically hard and soft particles. In such composites the coupling of the inner structure to the magnetic-field-induced changes of the properties is even more complicated than in MAEs containing one species of magnetic particles only, since the complex inner magnetic fields structure has to be evaluated and understood. For instance, when a remanent magnetization is induced in the hard magnetic component, magnetization and structuring of the soft magnetic components can as well take place even without an actively applied external field [8].

As it has been presented in [8], measurements of the first order reversal curves (FORCs) can be used as an effective method to study the magnetic interactions between the magnetically hard and soft particles inside the MAEs. The FORC method was firstly introduced by Roberts and Pike $[9,10]$ and provides a quantitative evaluation of the coercive field distribution and the local interaction field. The current work is an overview of the results obtained from the FORC measurements performed on the MAEs with mixed magnetically hard and soft components, as well as on the specimens containing magnetically hard particles only. In this context, the influence of various parameters, such as matrix stiffness particle ratio and training effect on the magnetization behavior of the hybrid material is presented. Furthermore, to understand the complex correlation between magnetization behavior and internal particle structure of MAEs, it is necessary to obtain a detailed view to the internal microstructures at different points in time of the FORC analysis. This overview also briefly addresses the microstructure analysis of the magnetized MAEs by X-ray computed microtomography, which provides important information about the relative structure and movements of the particles in relation to each other through a three-dimensional visualization of the particles. It assists in the interpretation of FORC analysis.

\subsection{Samples and methods}

\subsubsection{Sample preparation}

The MAE samples considered in this paper are based on a polydimethylsiloxane (PDMS) matrix. Usually a two-component composition with an organometallic crosslinking agent (Pt catalysts) is used. Tuning of the matrix stiffness is achieved by different mixing ratios of the individual polymer components and by dilution of the 
composition with a silicone oil. Details on a manufacturing processes are given elsewhere [5]. In addition particle-matrix-systems with an inelastic epoxy resin matrix are used as reference samples.

Magnetically hard NdFeB-alloy microparticles or combinations of hard magnetic NdFeB-alloy microparticles with soft magnetic carbonyl iron microparticles are used as filling components. The NdFeB-alloy particles have either a spherical (MQP-S-11 from Magnequench) or a flake-like shape (MQA-38-14 from Magnequench), with a size distribution of the spherical particles between 5 and $50 \mu \mathrm{m}$ and a length distribution of the flake-like particles between 10 and $100 \mu \mathrm{m}$ [11]. Before adding to the noncrosslinked matrix, the NdFeB particles are modified with a mixture of volatile alcohol and silicone oil to improve adhesion to the polymer chains [5]. The mean diameter of the precoated by the manufacturer spherical carbonyl iron particles (BASF, grade CC) is $\sim 5 \mu \mathrm{m}$.

The particles are added before the crosslinking reaction and distributed homogeneously during polymerization using mechanical stirring. The total particle content as well as the ratio of hard magnetic to soft magnetic components is varied for different samples as given in $[8,11]$.

\subsubsection{FORC method}

Magnetic characterization of MAEs was performed using vibrating sample magnetometers (VSM) of 7407 series from Lake Shore. All measurements have been conducted at room temperature. The magnetization behavior of the MAEs containing hard magnetic components used in this work was characterized using the FORC method.

The FORC method divides the measuring process (see Figure 10.1) into the following steps: (1) possibly training of the MAE sample by multiple repetition of the full magnetization cycle, (2) magnetization of the sample in a maximum positive field, (3) reduction of the magnetic field until the reverse field $H_{r}$ is reached, (4) measurement of a FORC curve, (5) $n$-fold repetition of the steps (2) - (4) with regular intervals between $H_{r}$ to $H_{r}=-H_{\text {max }}$. Set of the reversal magnetization curves obtained in this way is exemplary demonstrated in Figure 10.1. The resulting set of functions $M\left(H_{a}, H_{r}\right)$ provides the magnetization of the examined MAE at any point depending on the respective reverse field $H_{r}$ and the respectively applied magnetic field $H_{a}$. The FORC distribution is defined by the mixed derivation of the functional group according to $H_{r}$ and $H_{a}$

$$
\mu\left(H_{r}, H_{a}\right)=-\frac{1}{2} \frac{\partial^{2} M\left(H_{r}, H_{a}\right)}{\partial H_{r} \partial H_{a}}
$$

and the FORC diagrams are in turn the result of a coordinate transformation by $45^{\circ}$,

$$
\begin{aligned}
& H_{c}=\frac{H_{a}-H_{r}}{2} \\
& H_{u}=\frac{H_{a}+H_{r}}{2}
\end{aligned}
$$


so that the horizontal axis now corresponds to the coercive field $H_{c}$ and the vertical axis to the interaction field $H_{u}$. The FORCinel analysis software with a smoothing factor of 10 was used for the data treatment and calculations of FORC diagrams. In the measurements according to [8], 699 FORC curves were recorded based on the original algorithm of the MicroMag ${ }^{\mathrm{TM}}$ software of the Princeton Measurements Corporation. Further measurements include 250-350 curves based on an algorithm provided by the Lake Shore Software. The measuring time per point was between 1 and $2 \mathrm{~s}$ in all experiments.

\subsubsection{Microstructure analysis}

Computed X-ray microtomography $(\mu \mathrm{CT})$ is a nondestructive method for analyzing the internal microstructure of MAEs [12]. Paired with modern algorithms of digital image processing, the high resolution $\mu \mathrm{CT}$ makes it possible to differentiate individual particles and to track their movements under the influence of magnetic fields as demonstrated in Figure 10.2 [13-15]. The microstructural analysis performed using $\mu \mathrm{CT}$ is attended to assists in the interpretation of FORC diagrams.

\subsection{Results}

\subsubsection{Training effects}

The so-called training effect of magnetoactive elastomers with magnetically hard, flake-shaped NdFeB alloy particles has been investigated in [11]. During the magnetization of the samples it was observed that the first magnetization curve of a virgin

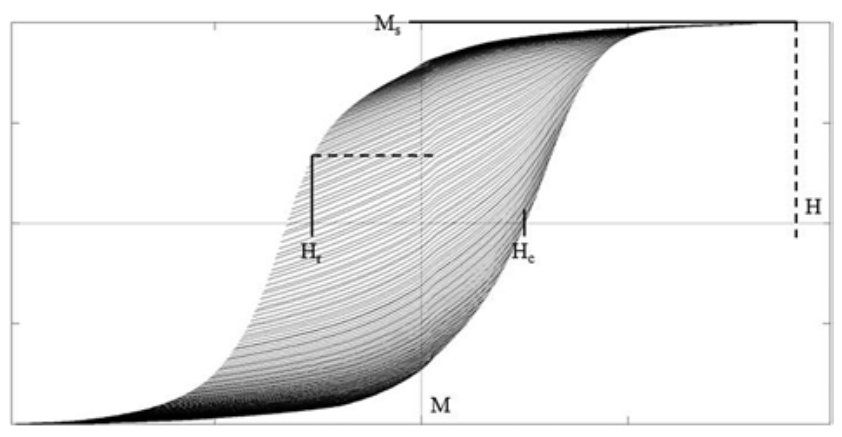

Figure 10.1: Function set of the reversal curves of an MAE with hard magnetic NdFeB particles and hard elastic matrix represented as magnetization $M$ over the applied magnetic field $H\left(H_{a}\right)$. A reversal or FORC curve starts at $H_{r}$ and runs to $H_{\text {max }}\left(\right.$ or $\left.H_{\text {Sat }}\right)$. The saturation magnetization $M_{S}$ is reached when more than $95 \%$ of the magnetic particles are magnetized. $H_{c}$ (coercive field) indicates the particles own magnetic field which remains after external magnetization. 

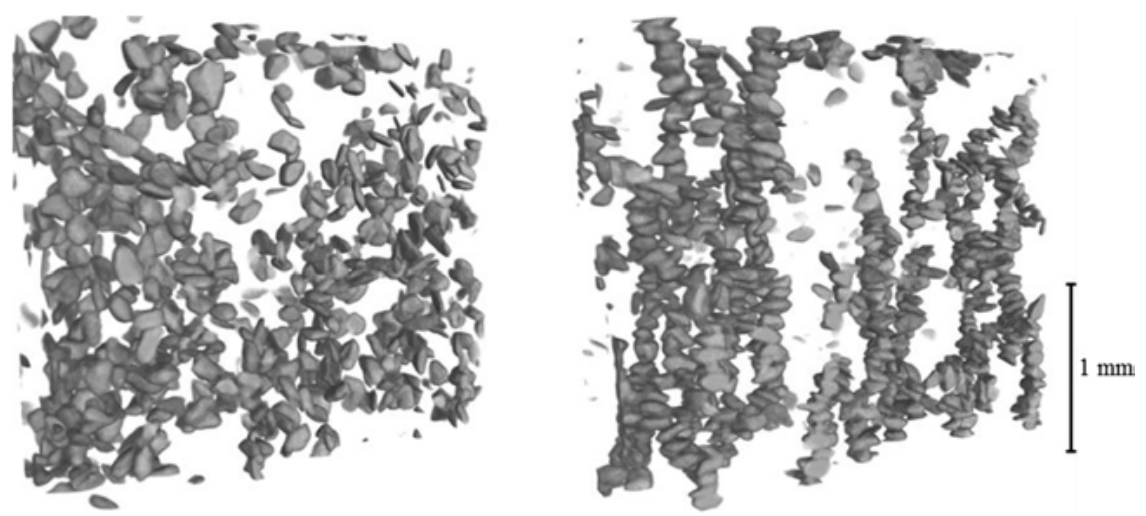

Figure 10.2: On the left side the $\mu \mathrm{CT}$ scan of a sample MAE with flake-like NdFeB-alloy particles before magnetization is shown. The particles are evenly distributed. The right side, in contrast, shows the scan of the same sample after it was magnetized at $2 \mathrm{~T}$. Now the particles have shifted within the soft elastic matrix and chain-like structures have formed [14, 15].

sample differs significantly from the following curves. By stepwise magnetization of the sample with $\mu \mathrm{CT}$ scans in between, it was possible to follow the particle movements and the alignment of their main axes relative to the magnetic field direction, as it is show in Figure 10.3. From the angular distribution of the particles, which was evaluated from the $\mu \mathrm{TC}$ scans at the different magnetization stages, it was possible to conclude that the particle-matrix coupling in the first magnetization loop is strong and only a part of the particles rotate. Only after further magnetization loops and the saturation of the sample the particles were able to rotate reversibly and align themselves along the magnetic field. As a consequence of the $\mu \mathrm{CT}$ scans with the corresponding angular distributions and the simulation model introduced in, a first, indirect impression of the irreversible matrix deformation behavior was obtained.

In $[17,18]$ the phenomena of training of MAE with hard magnetic spherical NdFeB-alloy particles were also analyzed experimentally and theoretically. According to this, complex magnetization processes of the individual grains take place in the multigrain particles, which interact with each other. The training effect is based on the fact that the magnetic moments within the individual grains change their orientation along the anisotropy axis during the magnetization loops and switch moments in some of the grains. The more often the magnetization loop is passed through, the more stable the system becomes since grains turn by the same angle under the influence of the magnetic field, and the same groups of grains begin to switch their magnetic moment. The magnetization of microparticles thus depends on the strength of the applied magnetic field as well as on the number of grains and does not correspond to the saturation magnetization of NdFeB-alloy powder, which is about 7.3 $\mathrm{T}[17,18]$.

Figures 10.4-10.6 demonstrate influence of the training effect on the FORC distribution obtained for the MAE with hard magnetic spherical NdFeB-alloy particles. The 


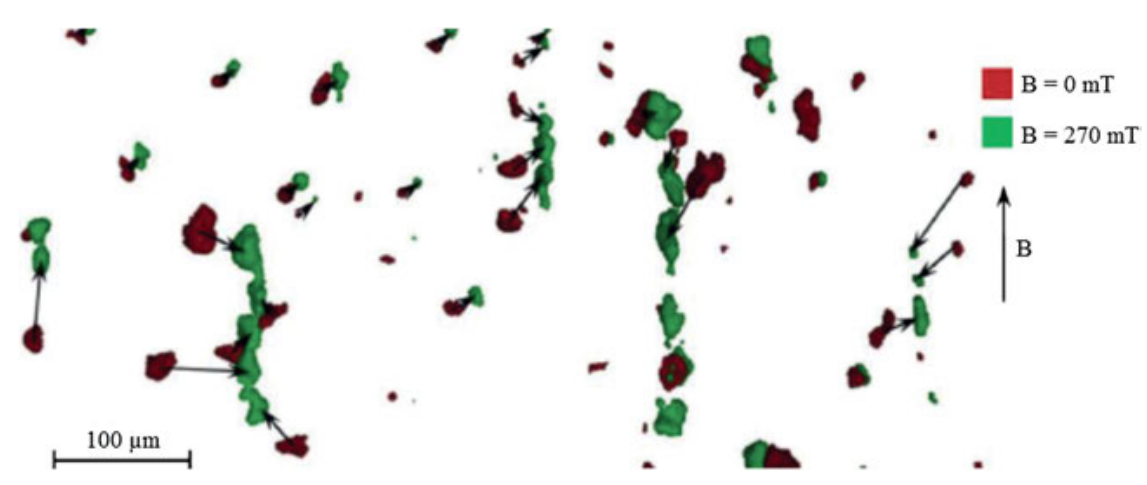

Figure 10.3: Combined $\mu \mathrm{CT}$ scans of a MAE with flake-shaped NdFeB-alloy particles, with and without an applied magnetic field, showing the movements and rotations of the particles by single particle tracking $[15,16]$.

sample has a soft elastic PDMS matrix $(E \sim 50 \mathrm{kPa})$ and filled with 10 vol\% of particles having a size of 5-20 $\mu \mathrm{m}$. In Figure 10.4 the FORC distribution of a sample which was not premagnetized before the FORC measurement is presented, while Figure 10.5 shows the results from a sample which was initially magnetized (from $H=0$ to $H_{\max }$ ) and then passed through a magnetization loop. In contrast in Figure 10.6 a sample passing through six magnetization loops was used for FORC measurement. From the FORC diagrams it can be seen that the peak of the coercivity field in the untrained sample is relatively large due to the still strong particle-matrix interactions. As the magnetization state of the system becomes increasingly stable, analogous to [17], the magnetization becomes larger and the peak of the coercivity field narrower. The particles can rotate reversibly in the meanwhile stable particle-matrix-system and show comparable movement patterns when a magnetic field is applied again (e.g., during further FORC measurements).

\subsubsection{Matrix influence}

The elasticity of the polymer matrix has a significant influence on the magnetization behavior of magnetoactive elastomers [19]. The more soft the matrix is, the more the particles can move within it under the influence of the applied magnetic field. Various particle-matrix MAEs containing magnetic hard particles were considered using FORC analysis. Corresponding results are shown in Figures 10.7 and 10.8.

Figure 10.7 shows that the central peak moves to the right along the coercive field axis with increasing modulus of elasticity. This drastic shift can be explained by the increasing restriction of particle movement. The stiffer the matrix, the less the particles can move and rotate. After all, in completely inelastic matrices, only inner-particle domain processes can take place in response to an external magnetic field. The interaction between the different particles type is also restricted with increasing matrix 

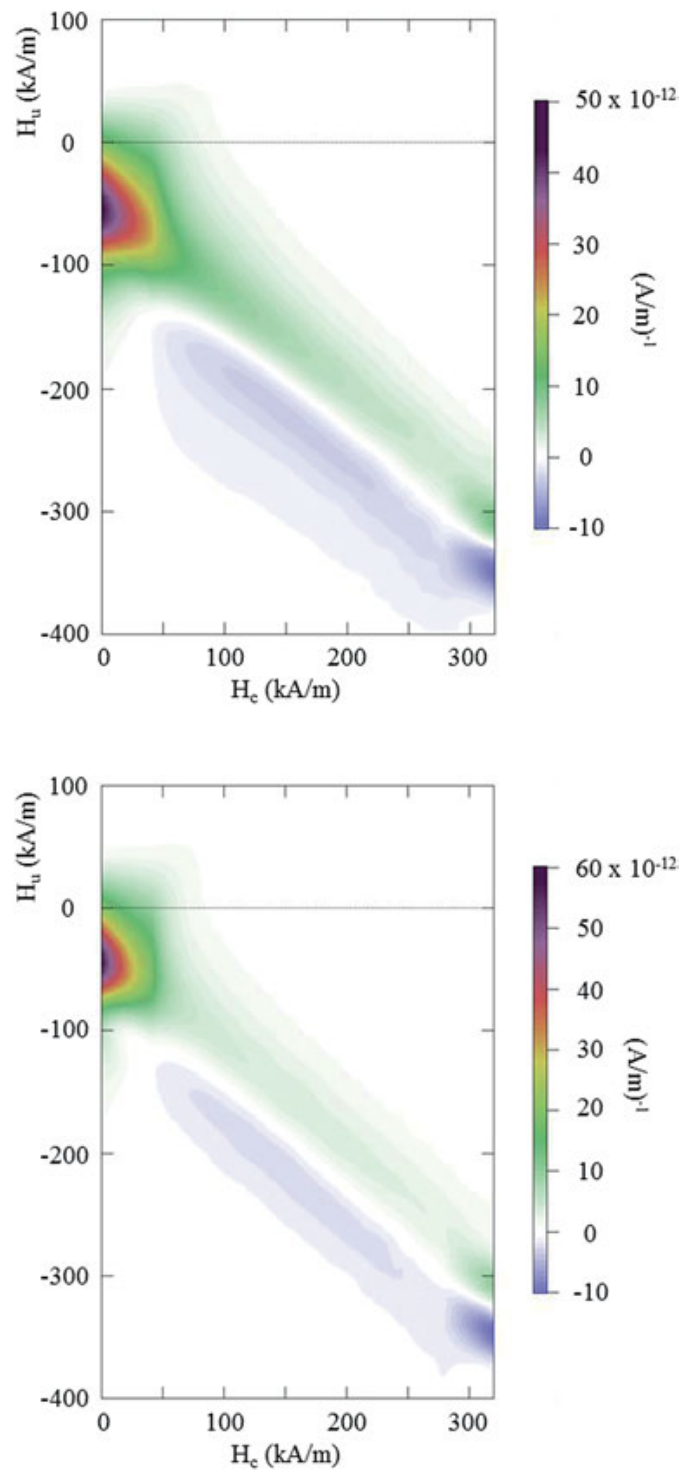

Figure 10.4: FORC diagram of an untrained sample of MAE with $10 \mathrm{vol} \%$ NdFeB particles $(<20 \mu \mathrm{m})$ in soft elastic matrix $(E \sim 50 \mathrm{kPa})$.

Figure 10.5: FORC diagram of MAE with 10 vol\% NdFeB particles $(<20 \mu \mathrm{m})$ in soft elastic matrix $(E \sim 50 \mathrm{kPa})$, sample was premagnetized one time.

stiffness. The narrow ridge along the interaction axis represents the coupling between reversible magnetization of the soft magnetic iron particles and the irreversible magnetization state of the entire system. The diagonal feature in the FORC diagrams, however, indicates magnetostatic coupling and the movement of the iron particles in the matrix as discussed in [8].

FORC measurement performed on specimens solely based on magnetic hard powder confirmed the significant influence of matrix elasticity on the movement behavior of the particles under the influence of magnetic fields (Figure 10.8). The stiffer 


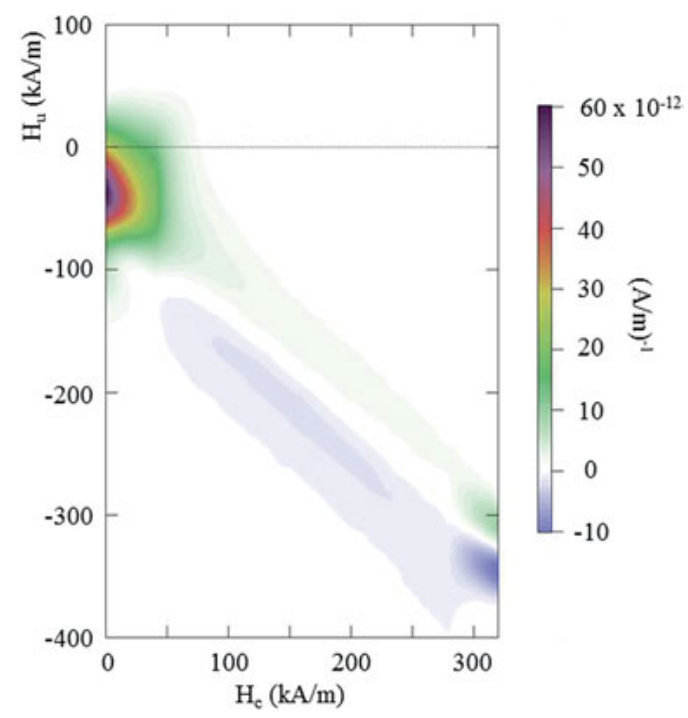

Figure 10.6: FORC diagram of MAE with 10 vol\% NdFeB particles $(<20 \mu \mathrm{m})$ in soft elastic matrix $(E \sim 50 \mathrm{kPa})$, sample was premagnetized six times.
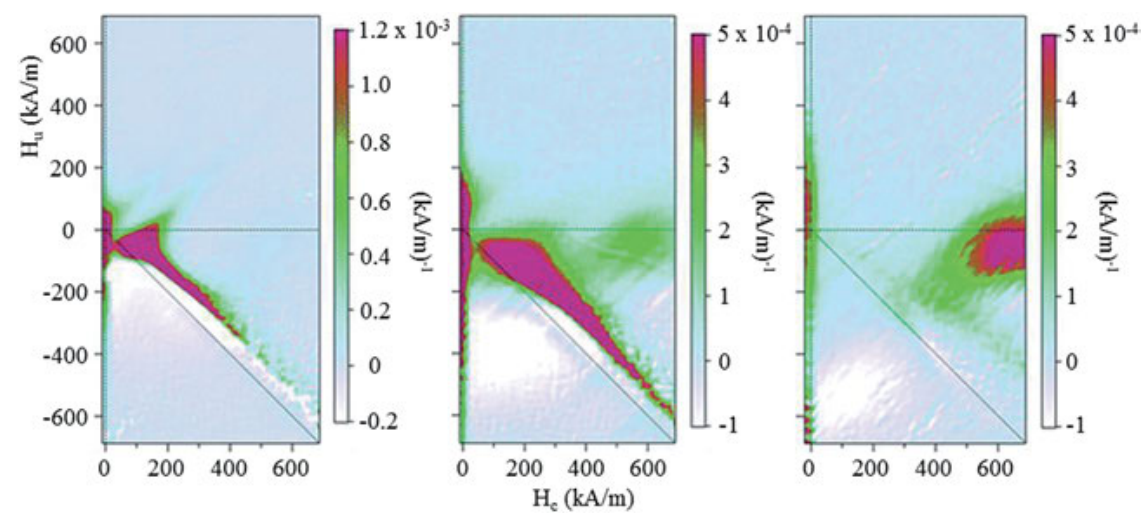

Figure 10.7: FORC diagrams of MAE with mixed magnetic filler components at a total particle content of $28 \mathrm{vol} \%$ and a mixing ratio of $\mathrm{NdFeB}$ particles to iron particles of $55: 45$. The elasticity of the polymer matrix increases from left to right: $50 \mathrm{kPa}$ (left), $120 \mathrm{kPa}$ (middle) and $440 \mathrm{kPa}$ (right) [8].

the matrix system is, the less the particles can rotate and move. Inner-particle domain processes increase, the magnetization of the system decreases. In the hard epoxy matrix the particles are firmly anchored which means that the particles can no more rotate, and the magnetization of the system is based entirely on the inner-particle processes of the multidomain particles, whose individual regions align themselves along the magnetic field lines or, according to [17], switch over at sufficiently high fields. In addition, a weak diagonal ridge is visible in the FORC diagrams obtained for the samples without magnetic soft powder. According to [20], this could be due to soft 


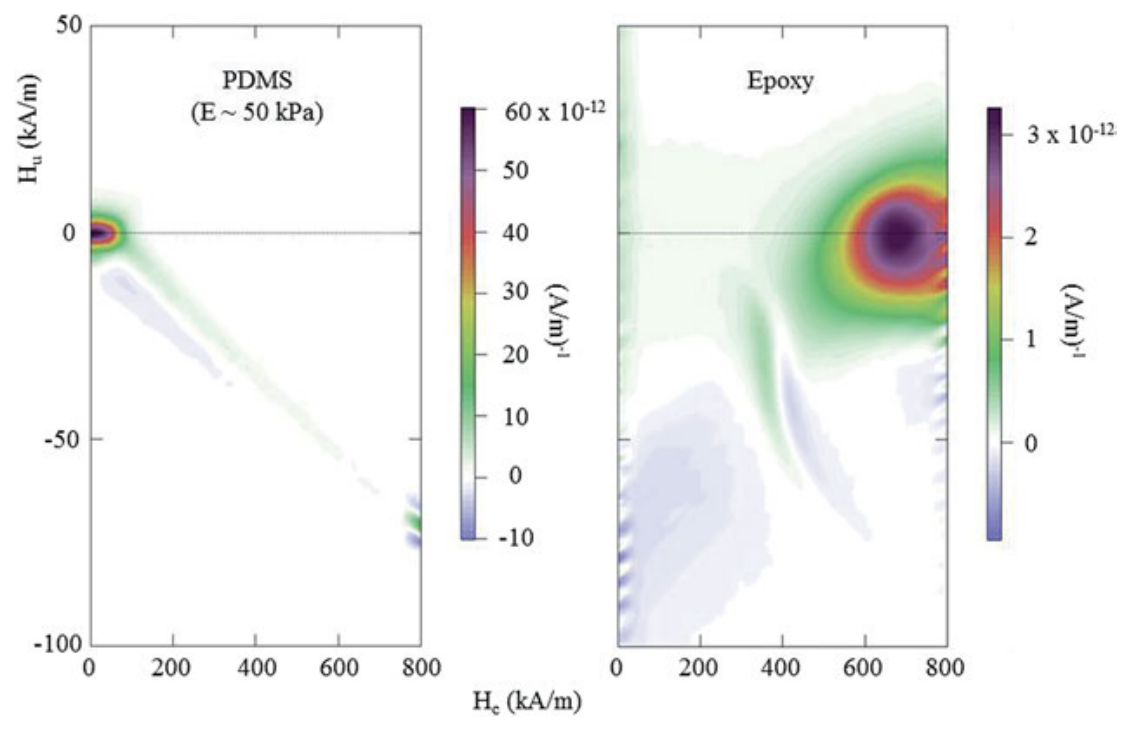

Figure 10.8: FORC diagrams of MAE with exclusively hard magnetic filling components at a particle content of 5 vol\%. The samples are trained before passing FORC measurement.

magnetic fractions in the magnetically hard NdFeB particles, but this has not been proven, so the origin of this FORC feature is still to be investigated.

\subsubsection{Particles influence}

\subsubsection{Particle ration}

The influence of the ratio of the mixed magnetic filling components on the magnetization behavior of the MAE was investigated in [8] to evaluate whether there are interactions between the different particle types that have a possible influence on the material properties. For this purpose, MAEs with a constant total particle content of 28 vol\% were used, but the ratio of magnetically hard NdFeB-alloy particles to magnetically soft carbonyl iron particles was varied. Figure 10.9 shows that as the iron particle content increases, the intensity of the irreversible features decreases while the reversible portions increase. The asymmetric degree along the $H_{u}$ axis results from the coupling of the reversible magnetization behavior of the iron particles and the irreversible magnetization state of the whole system. In contrast, the areas of irreversible magnetization that occur at interaction fields of $H_{u}<-50 \mathrm{kA} / \mathrm{m}$ are caused by the magnetostatic coupling and the movement of the particles within the matrix. In the case of higher contents of magnetically soft carbonyl iron particles, there are furthermore effects with positively and negatively applied fields. In the negative range, these are probably caused by a magnetization reverse of iron particles in the immediate 

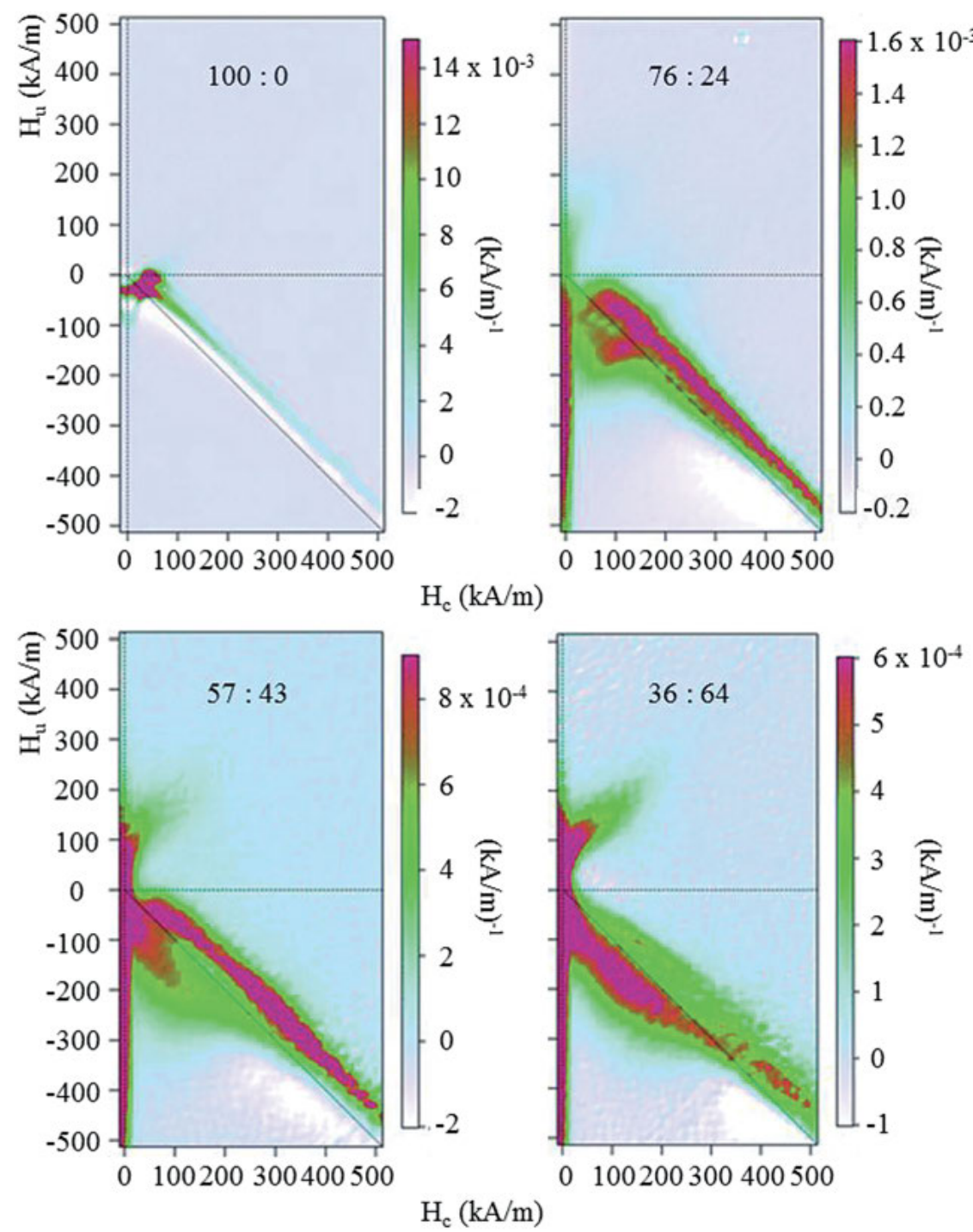

Figure 10.9: FORC diagrams of MAE with mixed magnetic filling components at a total particle content of $28 \mathrm{vol} \%$, a constant matrix stiffness and a varied mixing ratio of NdFeB-alloy particles to iron particles. For example, the FORC diagram at the top on the left side has a ratio of $100 \% \mathrm{NdFeB}$ particles to $0 \%$ Fe particles. The ratios of the other three diagrams are obtained in analogy $(76: 24$, $57: 43,36: 64)[8]$.

vicinity of positively magnetized NdFeB-alloy particles. The magnetization of these soft magnetic particles can only be reversed at this point by a negative field that compensates the positive remanent field of the nearby NdFeB-alloy particles. The effect in 


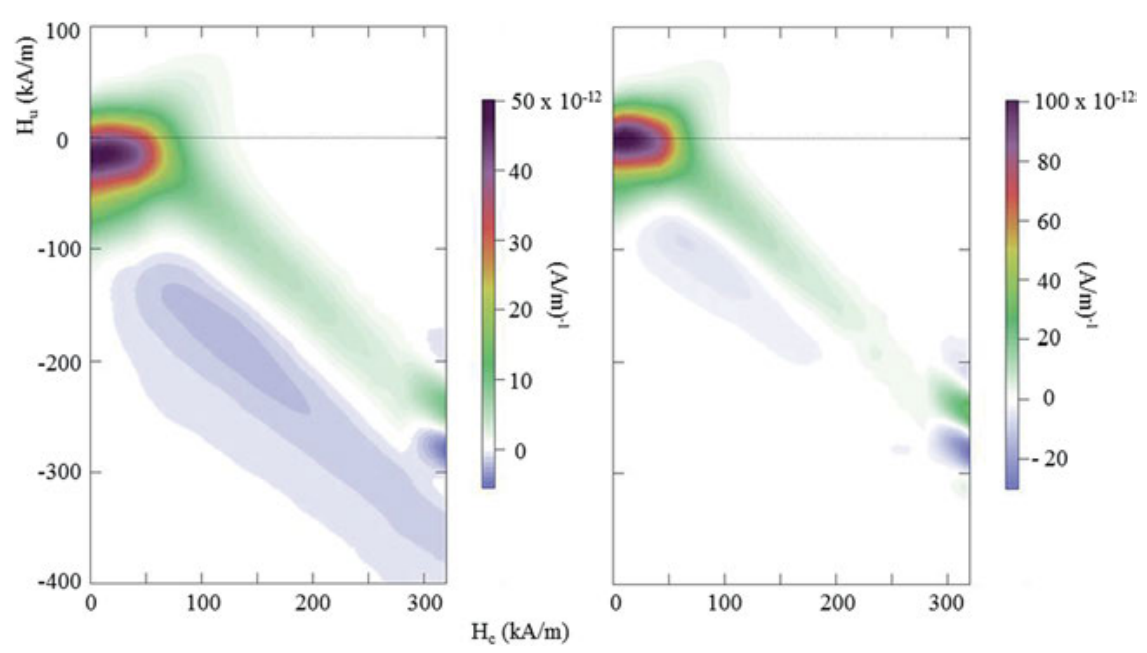

Figure 10.10: FORC diagrams of MAE with 10 vol\% hard magnetic NdFeB particles in a polymer matrix with an elasticity of $E \sim 50 \mathrm{kPa}$ and varying particle size distribution: 20-39 $\mu \mathrm{m}$ (left) and 63-79 $\mu \mathrm{m}$ (right). Both samples were trained in advance.

the positive region probably comes from the movement and restructuring of the iron particles in the matrix.

\subsubsection{Particle size}

According to the manufacturer's specifications the diameter of the spherical NdFeBalloy particles used by Magnequench is between 5 and $100 \mu \mathrm{m}$. To analyze the influence of particle size on the magnetic behavior of a composite, the particles were fractionated into different size ranges by the dry sieve method. Therefore the HAVER EML 200 Premium Remote Test Sieve Shaker with different nominal sizes of test sieves was used to fractionize the particles. FORC diagrams obtained for two samples with soft-elastic PDMS matrix $(E \sim 50 \mathrm{kPa})$ but different particle size classes are compared in Figure 10.10. The larger particles show a much higher magnetization, which may be explained by the correspondingly higher number of domains within the multigrain particles. Furthermore, the feature corresponding to the coercive field is larger in the FORC diagram obtained for the sample with the smaller particles. This can be explained by a more tightly structured particlepolymer network, which, in analogy to Figure 10.8, is accompanied by an increase in the coercive field. In relation to the larger $\mathrm{NdFeB}$ alloy particles, the smaller particle fraction has higher total surface area, which potentially allows more rubbers between coated particles and polymer chains as discussed in [21]. However, the exact reasons for the different behavior is not clear and still need to be investigated. 


\subsection{Conclusion and outlook}

Magnetoactive elastomers with magnetically hard particles or mixed magnetic filling components show a complex magnetization behavior that is influenced by several factors. This paper provides an overview of the influencing parameters for particlematrix systems with primary spherical NdFeB-alloy particles. The magnetic characterization is performed using the FORC method. While the training effect has already been demonstrated in the magnetization curves of such systems with magnetically hard NdFeB-alloy particles $[11,17,18]$, its influence on the FORC distributions is discussed in this study. The FORC diagrams also show that the magnetization behavior depends on the training state of the sample. While the particle-matrix-coupling is at first much more intense in the untrained state, the particles are able to move better with each magnetization cycle until they can finally rotate reversibly. These observations indirectly indicate irreversible damage to the polymer structure as a result of training a MAE sample.

The polymer matrix also has a significant influence on the movement behavior of the NdFeB-alloy particles and therefore on their magnetization characteristics. Especially in soft elastic polymers, reversible rotation and restructuring of the particles under the influence of an applied magnetic field is possible. In this case, the magnetization of the system results from the orientation of the particles in the field as well as from interparticle-interactions. In contrast, in stiff matrix-systems, such as in an inelastic epoxy matrix, the particles are firmly anchored in the matrix structure and the relatively small magnetization of the material compared to the previous case results exclusively from particle-internal domain effects. The coercive field also increases drastically with stiffer matrices.

Furthermore, NdFeB-alloy particles of different sizes in the micrometer range show different magnetic characteristics. As expected, composites with smaller particles have lower magnetization and slightly higher coercivity. This can be reasoned with a more distinct particlematrix-bond. The influence of the particle size is of particular importance with regard to future technical applications, since a constantly reproducible magnetization behavior of the particlematrix-system is required for series products.

As a consequence of the knowledge obtained to date on the magnetization behavior of magnetically active elastomers with hard magnetic NdFeB-alloy particles, a detailed examination of the relationship between magnetization properties and particle movements is required. This can be realized using a coupling of $\mu C T$-Scans to obtain the three-dimensional representation of the particle structures with the results of stepwise FORC measurements to obtain respective macroscopic response of the material. The results of such investigations are of particular importance for the development and verification of theoretical models, which in turn must be used for a wide range of technical applications, e.g., in sensor technology [22-24]. 
Author contributions: All the authors have accepted responsibility for the entire content of this submitted manuscript and approved submission.

Research funding: Financial support by Deutsche Forschungsgesellschaft (DFG) under Grant SO 18/24-1 within SPP 1681 and DFG-RFBR PAK 907 is gratefully acknowledged. Conflict of interest statement: The authors declare no conflicts of interest regarding this article.

\section{References}

1. Odenbach S. Microstructure and rheology of magnetic hybrid materials. Arch Appl Mech 2016;86: 269-79.

2. Odenbach S. Magnetic hybrid materials. Arch Appl Mech 2019;89:1-2.

3. Stepanov GV, Borin DY, Raikher YL, Melenev PV, Perov NS. Motion of ferroparticles inside the polymeric matrix in magnetoactive elastomers. J Phys Condens Matter 2008;20:204121.

4. Borin DY, Stepanov GV, Odenbach S. Tuning the tensile modulus of magnetorheological elastomers with magnetically hard powder. J Phys Conf 2013;412:012040.

5. Borin DY, Stepanov GV, Dohmen E. Hybrid magnetoactive elastomer with a soft matrix and mixed powder. Arch Appl Mech 2019;89:105-17.

6. Borin DY, Stepanov GV, Dohmen E. On anisotropic mechanical properties of heterogeneous magnetic polymeric composites. Phil Trans Math Phys Eng Sci 2019;377:20180212.

7. Borin DY, Stepanov GV. Magneto-mechanical properties of elastic hybrid composites. Phys Sci Rev 2020, (submitted to this issue).

8. Linke JM, Borin DY, Odenbach S. First-order reversal curve analysis of magneto-active elastomers. RSC Adv 2016;6:100407-16.

9. Roberts AP, Pike CR, Verosub KL. First-order reversal curve diagrams: a new tool for characterizing the magnetic properties of natural samples. J Geophys Res 2000;105:28461-75.

10. Roberts AP, Heslop D, Zhao X, Pike CR. Understanding fine magnetic particle systems through use of first-order reversal curve diagrams. Rev Geophys 2014;52:557-602.

11. Sánchez PA, Gundermann T, Dobrosedova A, Kantorovich S, Odenbach S. Importance of matrix inelastic deformations in the initial response of magnetic elastomers. Soft Matter 2018;14: 2170-83.

12. Borin DY, Günther D, Hintze C, Heinrich G, Odenbach S. The level of cross-linking and the structure of anisotropic magnetorheological elastomers. J Magn Magn Mater 2012;324:3452-4.

13. Schümann $M$, Odenbach $S$. The microstructure of magnetorheological materials characterized by means of computed X-ray microtomography. Phys Sci Rev 2020, (submitted to this issue).

14. Schümann M, Borin DY, Huang S, Auernhammer GK, Müller R, Odenbach S. A characterization of the magnetically induced movement of NdFeB-particles in magnetorheological elastomers. Smart Mater Struct 2017;26:095018.

15. Schümann M, Gundermann T, Odenbach S. Microscopic investigation of the reasons for field dependent changes of the properties of magnetic hybrid materials using X-ray micro tomography. Arch Appl Mech 2019;89:77-89.

16. Gundermann T, Odenbach S. Investigation of the motion of particles in magnetorheological elastomers by X- $\mu C T$. Smart Mater Struct 2014;23:105013.

17. Vaganov MV, Borin DY, Odenbach S, Raikher YL. Training effect in magnetoactive elastomers due to undermagnetization of magnetically hard filler. Physica B Phys Condens Matter 2020;578:411866. 
18. Vaganov MV, Borin DY, Odenbach S, Raikher YL. Mesomagnetomechanics of hybrid elastomer composites: magnetization of elastically trapped particles. J Magn Magn Mater 2019;499:166249.

19. Krautz M, Werner D, Schrödner M, Funk A, Jantz A, Popp J, et al. Hysteretic behavior of soft magnetic elastomer composites. J Magn Magn Mater 2017;426:60-3.

20. Chen P-A, Yang C-Y, Chang S-J, Lee M-H, Tang N-K, Yen S-C, et al. Soft and hard natures of Nd2Fe14B permanent magnet explored by first-order-reversal-curves. J Magn Magn Mater 2014;370:45-53.

21. Fu S-Y, Feng X-Q, Lauke B, Mai Y-W. Effects of particle size, particle/matrix interface adhesion and particle loading on mechanical properties of particulate-polymer composites. Compos B Eng 2008;39:933-61.

22. Becker TI, Zimmermann K, Borin DY, Stepanov GV, Storozhenko PA. Dynamic response of a sensor element made of magnetic hybrid elastomer with controllable properties. J Magn Magn Mater 2018;449:77-82.

23. Chavez J, Böhm V, Becker TI, Gast S, Zeidis I, Zimmermann K. Actuators based on a controlled particle-matrix interaction in magnetic hybrid materials for applications in locomotion and manipulation systems. Phys Sci Rev 2020, (submitted to this issue).

24. Becker TI, Raikher YL, Stolbov OV, Böhm V, Zimmermann K. Magnetoactive elastomers for magnetically tunable vibrating sensor systems. Phys Sci Rev 2020, (submitted to this issue). 